\title{
Detecting the Presence of Methicillin-Resistant Staphylococcus aureus on Acne Extractor used by Some Beauty Salons in Jatinangor
}

\author{
Ng Jo Sheng, ${ }^{1}$ Gita Indah Triyanti Rukmana, ${ }^{2}$ InneArline Diana ${ }^{3}$ \\ ${ }^{1}$ Faculty of Medicine Universitas Padjadjaran, ${ }^{2}$ Department of Microbiology and Parasitology \\ Faculty of Medicine Universitas Padjadjaran, ${ }^{3}$ Department of Dermato-Venerology Faculty of \\ Medicine Universitas Padjadjaran/Dr. Hasan Sadikin General Hospital, Bandung
}

\begin{abstract}
Background: Over the past few decades, methicillin-resistant Staphylococcus aureus (MRSA) has become a major cause of nosocomial infection and has recently emerged in the community. Community associated MRSA (CA-MRSA) have grown drastically over the years and have mostly manifested as a form of skin infection. The MRSA infection usually occurs in individuals with skin lesions serving as a site of entry for the bacteria. The aim of this study was to determine if MRSA is presence on the acne extractor used by some beauty salons in Jatinangor.

Methods: A total of 40 samples were taken from 14 beauty salons in Jatinangor from September to October 2013. The samples were then cultured on mannitol salt agar. The isolated colonies were then gram stained, followed by catalase and coagulase test. The colonies having positive for Staphylococcus aureus (S. aureus) were then tested for cefoxitin susceptibility.

Results: Overall 3 of the isolates were positive for $S$. aureus and none were positive for MRSA.

Conclusions: Only a small fraction of the isolates are positive for $S$. aureus and no MRSA is detected.. [AMI.2017;4(1):64-8]
\end{abstract}

Keywords: Acne extractor, beauty salons, community associated MRSA (CA-MRSA)

\section{Introduction}

Staphylococcus aureus (S. aureus) is part of the Staphylococcacceae group, and it is the most frequently seen of the staphylococci strain. The normal habitats of $S$. aureus are the human skin and mucosal surfaces. Over time, the staphylococcal infections have become increasingly difficult to treat. This is due to the $S$. aureus development of resistance towards antibiotics. One of the most commonly encountered forms of resistant $S$. aureus is the methicilin-resistant Staphylococcus aureus (MRSA). ${ }^{1,2}$ Methicilin-resistant Staphylococcus aureus infections are more severe as compared to $S$. aureus infection, and are highly associated with vulnerable populations. Even more recently, community acquired MRSA infections are commonly seen in healthy individuals. These individuals usually present with cutaneous manifestations. ${ }^{3}$ Based on the previous study conducted by Center of
Disease Control and Prevention (CDC) in year 2005 , there was an estimate of 94,000 cases of invasive life-threatening MRSA infection with about 19,000 deaths due to the MRSA infection. A 15-year study conducted by the Medical Center of San Diego from 1990 to 2004 showed that of the 1888 cases collected, 35\% were nosocomial acquired MRSA infection while the latter $65 \%$ were community acquired MRSA infection. ${ }^{4,5}$ The transmission of MRSA is usually through direct contact with an infected person, or a carrier and touching MRSA contaminated materials or substance..$^{5-7}$

According to "Outbreak of Invasive Methicillin-Resistant Staphylococcus aureus Infection Associated with Acupuncture and Joint Injection", data showed that all patients who were in contact with the MRSA colonized needle developed severe invasive MRSA infection. This study has shown that in a community setting, the use of invasive instrument increases the risk of developing

Correspondence: Ng Jo Sheng, Faculty of Medicine, Universitas Padjadjaran, Jalan Raya Bandung-Sumedang Km.21, Jatinangor, Sumedang, Indonesia, Phone: +6285759632537 Email: ngjosheng@gmail.com 
MRSA infection. ${ }^{6-9}$ In Jatinangor Public Health Centre (PHC), a study was conducted in 2012 whereby MRSA was detected from the isolates of hand swabs of the medical and paramedical staff, proving that there is nosocomial MRSA in PHC, Jatinangor. A beauty salon is an establishment that provides cosmetic treatment for both men and women. One of the common services offered by the beauty salon is facial treatment which involves the use of an acne extractor. The acne extractor is an invasive instrument, which in practice is used to remove comedones; thus, this serves as a portal of entry for MRSA to infect the individual. The study was conducted to determine if MRSA can be found on the acne extractor from some beauty salons in Jatinangor. As the acne extractor may serve as a reservoir for MRSA, there is higher possibility of colonization of MRSA there than any other equipment found in the beauty salons.

\section{Methods}

A descriptive laboratory cross-sectional study was conducted from September to October 2013 in Jatinangor sub district. Samples were obtained prior to cleaning, washing, sterilizing and applying to face from beauty salons which offered facial treatments and willing to participate. The samples were then tested in the Microbiology Laboratory of Faculty of Medicine Universitas Padjadjaran. The study conducted was approved by the Health Research Ethics Committee of Faculty of Medicine Universitas Padjadjaran.

The sample size was calculated using the descriptive study of dichotomous variable formula, and a total of 40 samples were used for the study. The samples were obtained from the acne extractor through convenience sampling technique and were then cultured on mannitol salt agar. After a 24-hour incubation period, the isolated colonies were gram stained. Colonies that are gram positive were then further tested for catalase enzyme using hydrogen peroxide $(10 \%)$, and then tested for coagulase using human plasma. Colonies that showed positive reactions were then further

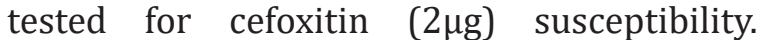
The diameter of the clear zones around the cefoxitin disc was measured using a ruler. The S. aureus was considered resistant if the clear zone was less than $22 \mathrm{~mm}$.

The beauticians of the salons were then asked to answer 2 questions about the technique of sterilization used in the salons. The data obtained from the study and the beauticians were then presented descriptively as incidence of MRSA and knowledge and awareness of sterilization protocol.

\section{Results}

The total number of beauty salons participated was 14 out of the 16 beauty salons along Jatinangor Street. Isolates are considered $S$. aureus positive when are proven to be Gram positive, with catalase and coagulase positive. The MRSA is considered presence when there is resistance to cefoxitin disc.

Based on the study, out of the 40 samples collected and tested, only 3 samples were found to be positive for $S$. aureus. After further microbiological test, none of the samples were positive for MRSA.

The awareness and performance was done to assess the ability of the beauticians to sterilize in an acceptable manner. The results showed that more than half of the beauticians were unable to sterilize well although they were aware of the standard sterilization technique (Table 1).

\section{Discussions}

The study was performed to detect the presence of MRSA on acne extractor used by some beauty salons along Jatinangor Street. As stated in the previous chapters, the MRSA was detected microbiologically, whereby the specimen collected is cultured, gram stained, tested for catalase and coagulase enzyme, and antibiotic susceptibility. As MRSA is a common bacteria of antibiotic resistance, it is chosen as the subject of the study. Besides, many studies conducted have shown that CA-MRSA, have a

Table Awareness and Performance of Proper Sterilization Technique for the Acne Extractor

\begin{tabular}{lcc}
\hline \multicolumn{1}{c}{ Standard Sterilization Technique } & $\begin{array}{c}\text { Performed well } \\
(\mathbf{n}=\mathbf{2})\end{array}$ & $\begin{array}{c}\text { Performed poorly } \\
(\mathbf{n}=12)\end{array}$ \\
\hline Knows $(\mathrm{n}=9)$ & 2 & 7 \\
Does not know $(\mathrm{n}=5)$ & 0 & 5 \\
\hline
\end{tabular}


widespread distribution. The sterility of the acne extractor also plays a major role in the presence of the bacteria.

A total of 40 samples were used in the study. The samples collected were swabs of the acne extractor from some beauty salons along Jatinangor Street. As of the 16 beauty salons along Jatinangor Street, only 14 beauty salons took part in the study. The other 2 beauty salons refused to participate due to worry of losing customers. The participants were given adequate information and procedure about the study before samples were collected. From the 40 samples obtained, no MRSA was found on the surface of the acne extractors. However, the results showed that there were 3 samples positive for $S$. aureus.

In this study, there was no MRSA found; thus, there was no CA-MRSA. Therefore, it can be said that the beauty salons along Jatinangor street were free of the CA-MRSA. The MRSA is usually transmitted through the direct or indirect contact with either contaminated instruments or infected patients who are involved in the community. Hence, there was no risk of MRSA infection for the clients of the beauty salons along Jatinangor Street.

Only 2 subjects out of the 14 subjects were able to sterilize the equipment in an acceptable manner. When the participants of the study were asked to demonstrate the technique of sterilization used, none of them used alcohol to sterilize their equipment. The 2 participants who passed this test did so by soaking the acne extractor in hot water. A study conducted shows that after practicing comprehensive infection control measures which involves sterilization and control will improve infection spread. ${ }^{10}$

Community associated MRSA studies conducted in America and Switzerland have shown increasing prevalence over the years. ${ }^{11,12}$ From the similar study conducted in America, community associated MRSA infection accounted for $12 \%$ of all the MRSA infection documented during the period of study. The study also shows that the MRSA is detected from the community originated from the healthcare setting. ${ }^{11}$ The main reason for the widespread of MRSA in the community and healthcare is due to the common use of $\beta$-lactam antibiotics prescribed by the primary care doctors. ${ }^{11}$ As discussed in previous chapters, the common presentation of the MRSA infection is skin and soft tissue infection. Besides, CA-MRSA is much more severe compared to nosocomial MRSA as it is resistant to more classes of antibiotics. ${ }^{11}$
From the results of the study, the acne extractor was contaminated with $S$. aureus; therefore, it serves as a source of infection. When the contaminated acne extractor is used to remove the comedones on the facial skin, the $S$. aureus may be able to penetrate the deep skin tissue through the puncture site. This will then develop into a $S$. aureus infection of the skin. Common presentation of the infection is furuncle or also known as a boil. When not treated adequately, the $S$. aureus can further penetrate the tissue and move deeper increasing the risk of developing invasive $S$. aureus infection. Examples of those diseases are bacteremia, endocarditis, metastatic infections, sepsis and toxic shock syndrome. ${ }^{13}$ In addition, the patients of $S$. aureus infection had the risk of $20-50 \%$ of developing MRSA infection. ${ }^{14}$

Despite the low percentage of $S$. aureus found, many other species of bacteria were found to be present on the acne extractor. Though the species of the bacteria were not identified, this shows that the acne extractor is not sterilized well. A few of the beauty salons had acne extractors that were already rusty but was still in use. The acne extractors were also not kept well by the beauticians; most of the acne extractor was kept together with hair brushes, hair combs, scissors and the other salon equipment. The beauticians admitted not to frequently clean the extractor and only do so with a wet cloth when it is used.

Only 2 samples out of the 14 samples were able to sterilize the equipment in an acceptable manner. None of the subjects knew of the use of alcohol as a sterilizing agent. This shows that proper sterilization is not performed in the beauty salons. Without proper sterilization, the acne extractor may serve as a breeding ground not only for $S$. aureus but also other bacteria. When the unsterilized acne extractor is used on clients after clients, the risk of transmission increases; and thus, it increases the risk of clients developing skin lesion infection of $S$. aureus origin.

Cross contamination also takes place when the acne extractor is kept in an unhygienic and unsterilized condition. The acne extractor will then harbor many types of bacteria including the $S$. aureus. As mentioned in the previous chapter, cross contamination serves as a risk of developing much more severe $S$. aureus infections.

All this can be easily prevented by hygienic steps before, during and after the use of acne extractor. Previous studies have shown that using alcohol alone is adequate 
in killing bacteria and thus sterilizing the acne extractor. ${ }^{15}$ Therefore, it is definitely recommended to use alcohol to sterilize the acne extractor. Simple steps like this can ensure that the acne extractor is safe to be used. The knowledge of the beautician on sterilization technique is inadequate as there is no specific course and guideline to be followed by them.

The lack of awareness about sterilization puts the clients at risk of developing skin lesions. All this can be prevented easily with knowledge about sterilization and frequent practice of the sterilization technique.

Methicillin-resistant Staphylococcus aureus is an antibiotic resistant form of $S$. aureus which is much more difficult to treat. Although infection brought about by the MRSA is the same as $S$. aureus, treating the infection is much more difficult due to antibiotic resistance and fear of further antibiotic resistance. From the study, no community associated MRSA was found. This shows that the acne extractors in the salons are still free of the much more severe form of $S$. aureus. However, if no precaution is taken, the risk of developing MRSA on the acne extractor is high. Thus, it is important for the beauticians of the beauty salon to start practicing the standard sterilization protocol that is implemented worldwide, that is by using alcohol to clean all their equipment including the acne extractor.

There is no CA-MRSA found in beauty salons along Jatinangor street. There is however $S$. aureus and other species of bacteria found on the acne extractor from the beauty salons. This can all be accounted by the lack of awareness and practice of proper sterilization on the equipments including the acne extractor.

Throughout the study, there were a few limitations that were met. Among them are the changes in behavior of the participants of the study such as increased frequency of washing the instruments and also better hygienic practices. There is also the lack of participation of the beauty salon in the study due to worry of losing their customers.

In conclusion, based on the results of the study, it shows that there is no MRSA found on the acne extractor. However, the sterilization of the acne extractor is inadequate, and thus, it accounts for the presence of $S$. aureus and other unidentified species of bacteria. Therefore, it is important for the implementation of standard and proper handling and sterilization of the acne extractor. This also proves that there is no CA-MRSA.

For future study, it is needed to do random visitation to the salon and also to give better explanation to the participants to ensure more participation. It is also recommended that a larger sample size needs to obtain for covering the whole of Jatinangor sub district. Finally, the use of a questionnaire would be helpful in obtaining the beautician's knowledge on sterilization.

\section{References}

1. Brown DF, Edwards DI, Hawkey PM, Morrison D, Ridgway GL, Towner KJ, et al. Guidelines for the laboratory diagnosis and susceptibility testing of methicillin-resistant Staphylococcus aureus (MRSA). J Antimicrob Chemother. 2005;56(6):1000-18.

2. Klevens RM, Morrison MA, Nadle J, Petit $\mathrm{S}$, Gershman $\mathrm{K}$, Ray $\mathrm{S}$, et al. Invasive methicillin-resistant Staphylococcus aureus infections in the United States. JAMA. 2007;298(15):1763-71.

3. Boucher HW, Corey GR. Epidemiology of methicillin-resistant Staphylococcus aureus. Clin Infect Dis. 2008;46Suppl 5:S344-9.

4. Schooneveld T, Rupp M. Control of grampositive multidrug resistant organism. In: Lautenbach E, Woeltje KF, Malani PN, editors. Practical healthcare epidemiology. 3rd ed. London: University of Chicago Press; 2010. p. 197-204.

5. Schwartz B, Chambers H. Clinical manifestations of community acquired MRSA infections. In: Crossley KB, Jefferson KK, Archer GL, Fowler VG, editors. Staphylococci in Human Disease. 2nd ed. West Sussex: Blackwell Publishing; 2009. p. 531.

6. Hardy KJ, Oppenheim BA, Gossain S, Gao F, Hawkey PM. A Study of the relationship between environmental contamination with methicillin-resistant Staphylococcus Aureus (MRSA) and patients' acquisition of MRSA. Infect Control Hosp Epidemiol. 2006;27(2):127-32.

7. Al-Tawfiq JA. Father-to-infant transmission of community-acquired methicillinresistant Staphylococcus aureus in a neonatal intensive care unit. Infect Control Hosp Epidemiol. 2006;27(6):636-7.

8. Murray R, Pearson JC, Coombs GW, Flexman JP, Golledge CL, Speers DJ, et al. Outbreak of invasive methicillinresistant Staphylococcus aureus infection associated with acupuncture and joint injection. Infect Control Hosp Epidemiol. 2008;29(9):859-65. 
9. Rodriguez D. Acne Extraction. New York: Everyday Health Media, LLC; 2010 [updated 17/02/2010; cited 25 February 2013];Available from: http:// www.everydayhealth.com/acne/acneextraction.aspx

10. Yokoe DS, Classen D. Improving patient safety through infection control: a new healthcare imperative. Infect Control Hosp Epidemiol. 2008 ;29Suppl 1:S3-11.

11. Naimi TS, LeDell KH, Como-Sabetti K, Borchardt SM, Boxrud DJ, Etienne J, et al. Comparison of community-and health care-associated methicillin-resistant Staphylococcus aureus infection. JAMA. 2003;290(22):2976-84.

12. Harbarth S, François P, Schrenzel J, Fankhauser-Rodriguez C, Hugonnet S, Koessler T, et al. Community-associated methicillin-resistant Staphylococcus aureus, Switzerland. Emerg Infect Dis. 2005;11(6):962-5.

13. Liu C, Bayer A, Cosgrove SE, Daum RS, Fridkin SK, Gorwitz RJ, et al.Clinical practice guidelines by the infectious diseases society of America for the treatment of methicillin-resistant Staphylococcus aureus infections in adults and children. Clin Infect Dis. 2011;52(3):e18-55.

14. Huang SS, Platt R. Risk of methicillinresistant Staphylococcus aureus infection after previous infection or colonization. Clin Infect Dis. 2003;36(3):281-5.

15. Rauk PN. Educational intervention, revised instrument sterilization methods, and comprehensive preoperative skin preparation protocol reduce cesarean section surgical site infections. Am J Infect Control. 2010;38(4):319-23. 